\title{
A contribution to the lichen flora of North East Greenland
}

\author{
Hansen, Eric Steen
}

Published in:

Botanica Lithuanica

DOI:

10.2478/v10279-012-0013-7

Publication date:

2012

\section{Document version}

Publisher's PDF, also known as Version of record

Document license:

CC BY-NC-ND

Citation for published version (APA):

Hansen, E. S. (2012). A contribution to the lichen flora of North East Greenland. Botanica Lithuanica, 18(2), 109116. https://doi.org/10.2478/v10279-012-0013-7 


\title{
A CONTRIBUTION TO THE LICHEN FLORA OF NORTH EAST GREENLAND
}

\section{Eric Steen Hansen}

Natural History Museum of Denmark of Copenhagen University, Botanical Museum, Gothersgade 130, DK1123 Copenhagen, Denmark; e-mail: erich@snm.ku.dk

\begin{abstract}
Hansen E. S., 2012: A contribution to the lichen flora of North East Greenland [Papildomi duomenys apie šiaurès rytų Grenlandijos flora]. - Bot. Lith., 18(2): 109-116.

The paper lists 83 species of lichens from North East Greenland between $75^{\circ}$ and $80^{\circ} \mathrm{N}$. A little more than half of the species, viz. 45, were recorded for the first time from this region. Fell-field lichens from alkaline and neutral as well as slightly acid ground are presented in the material, which, however, also contains some species of lichens found growing in moist heath localities as well as those with snow patches. The material includes numerous saxicolous lichens from siliceous bird rocks and rocks without visible influence of guano including some alkaline rocks.
\end{abstract}

Keywords: lichens, Danmarkshavn, Greenland.

\section{INTRODUCTION}

Contrary to the extensive area between Scoresby Land $\left(\mathrm{c} .72^{\circ} \mathrm{N}\right)$ and Wollaston Forland $\left(\mathrm{c} .74^{\circ} \mathrm{N}\right)$, which was investigated lichenologically during two Norwegian expeditions in 1929 and 1930 (LyNGE \& Scholander, 1937; Lynge, 1940), the coastal area between $75^{\circ}$ and $80^{\circ} \mathrm{N}$ is relatively poorly known as regards lichens. The botanist of the Danmark Expedition (1906-1908), Andreas Lundager collected 68 species of lichens, in particular around Danmarkshavn $\left(76^{\circ} 46^{\prime} \mathrm{N}\right)$, which were determined by GALLøE (1910). Some of these species, for example, Cladonia foliacea and Lecidea fuscoatra appeared to be wrongly identified. Børge Lauritsen, who was a radiosonde-man at Danmarkshavn in the 1980 s, collected about a hundred specimens of lichens from different localities of the region. The material was handed over to the author for identification. The same applies to the lichen collection consisting of about 80 specimens and made up by the Danish botanists Christian Bay and Bent Fredskild in summer 1990. The above-mentioned material was collected from a number of localities situated to the north of Danmarkshavn. The two collections sup- plement each other fairly well. Totally they indicate rich lichen flora. In 1995, Vagn Alstrup, Fred Daniels and the author collected more than four hundred taxa of lichens in Kronprins Christian Land, north of the present investigation area, which include southernmost Lambert Land (AlstruP et al., 2000). The lichen flora of Central East Greenland, including the extensive Scoresby Sund area, which is in need of further lichenological investigation, has been treated by several authors (BRANTH, 1894; Hartz, 1895; Hansen, 1982, 2006, 2008a, b, 2009, 2010). The present paper aims to stimulate future lichen research in the region, which presumably is just as rich in lichens as, for example, Kronprins Christian Land.

\section{MATERIALS AND METHODS}

The present paper is based on the collections made up by Børge Lauritsen in 1981 and 1982 and by Christian Bay and Bent Fredskild in 1990. Lichens were collected at numerous sites along the coast from Godfred Hansens $\varnothing$ in Dove Bugt to the east coast of Lambert Land (the coordinates of the sites 
are given in the list of species; see also FREDSKILD \& BAY, 1991). A total of 195 lichen specimens were studied using Zeiss light microscopes and identified by the author. The nomenclature in the list follows SAnTESSON et al. (2004) when appropriate. The specimens are deposited at the Botanical Museum of the University of Copenhagen (C).

\section{CHARACTERISTICS OF THE STUDY AREA}

Collecting of lichens was carried out at the altitudes up to c. $200 \mathrm{~m}$ a. s. 1 . on islands and along the mountainous coast between $75^{\circ}$ and $80^{\circ} \mathrm{N}$. The mountains are mainly composed of Caledonian gneissis with darker, more or less basic intrusions (Escher \& Stuart Watt, 1976). More or less alkaline soil occurs in some areas, for example, Schnauders Ø, V. Clausen Fjord and Lambert Land.

Floristically and climatically, the investigation area covers from the medium arctic, continental zone (Godfred Hansens Ø, Mørkefjord) and the medium arctic, oceanic zone (Danmarkshavn) to the high arctic, oceanic zone (Lambert Land) (JENSEN, 1999). The vegetation types vary from open fell-fields and grassland slopes (Germania Land) to mixed dwarf shrub heaths dominated by Cassiope, Dryas, Salix and Vaccinium uliginosum (V. Clausen Fjord). A dry, steppelike vegetation with Carex supina, Calamagrostis purpurascens, Kobresia myosuroides and lichens with preference for alkaline soil such as Acarospora schleicheri occurs in the above-mentioned locality (FREDSKILD \& BAY, 1991). Dryas-Carex rupestris heaths rich in lichens are widely distributed on Lambert Land. The whole investigation area is characterized by arctic climate with long, extremely cold winters and short, cold summers. The mean annual precipitation is estimated to be maximum c. $300 \mathrm{~mm}$, but it is considerably lower in the continental areas. The mean annual temperature is lower than $-10{ }^{\circ} \mathrm{C}$ (HANSEN, 1982).

\section{RESULTS AND DISCUSSION}

\section{List of species}

An asterix $(*)$ in front of the name in the list of lichens indicates that the taxon is an addition to the lichen flora occurring in the region between $75^{\circ}$ and $79^{\circ} \mathrm{N}$ in East Greenland. The collections, distributed previously from Herbarium $\mathrm{C}$ as a part of Lichenes Groenlandici Exsiccati (LGE), are stated by their numbers.

* Acarospora schleicheri (Ach.) A. Massal. V. Clausen Fjord, $77^{\circ} 30^{\prime} \mathrm{N}, 20^{\circ} 34^{\prime} \mathrm{W}$, alt. $50 \mathrm{~m}$, on mineral soil, C. Bay \& B. Fredskild, 15 August 1990.

* Arctocetraria nigricascens (Nyl.) Kärnefelt \& Thell - Stormlandet, north of Depotnæs, $77^{\circ} 36^{\prime} \mathrm{N}$, $18^{\circ} 57^{\prime} \mathrm{W}$, alt. $50 \mathrm{~m}$, on soil, C. Bay \& B. Fredskild, 20 August 1990.

Brodoa oroarctica (Krog) Goward - Danmarkshavn, $76^{\circ} 46^{\prime} \mathrm{N}, 18^{\circ} 36^{\prime} \mathrm{W}$, on siliceous rock together with Lecidea atrobrunnea and Pseudephebe minuscula, B. Lauritsen, 25 March 1981.

* Bryocaulon divergens (Ach.) Kärnefelt - Danmarkshavn, $76^{\circ} 46^{\prime} \mathrm{N}, 18^{\circ} 36^{\prime} \mathrm{W}$, on soil together with Gowardia nigricans and Thamnolia vermicularis v. subuliformis, B. Lauritsen, 12 June 1981.

* Bryoria chalybeiformis (L.) Brodo \& D. Hawksw. - Germania Land, Flade Bugt, c. $77^{\circ} 15^{\prime} \mathrm{N}, 19^{\circ} 30^{\prime} \mathrm{W}$, alt. $210 \mathrm{~m}$, on soil, B. Lauritsen, 4 May 1981; Stormlandet, north of Depotnæs, $77^{\circ} 36^{\prime} \mathrm{N}, 18^{\circ} 57^{\prime} \mathrm{W}$, alt. $50 \mathrm{~m}$, on soil, C. Bay \& B. Fredskild, 20 August 1990; De Franske Øer, $78^{\circ} 37^{\prime} \mathrm{N}, 18^{\circ} 35^{\prime} \mathrm{W}$, alt. $125 \mathrm{~m}, \mathrm{C}$. Bay \& B. Fredskild, 5 August 1990.

* Buellia elegans Poelt - Nr. Mellemland, $78^{\circ} 30^{\prime} \mathrm{N}, 21^{\circ} 10^{\prime} \mathrm{W}$, on mineral soil, C. Bay \& B. Fredskild, 4 August 1990.

* Caloplaca castellana (Räsänen) Poelt - Nr. Mellemland, $78^{\circ} 30^{\prime} \mathrm{N}, 21^{\circ} 10^{\prime} \mathrm{W}$, on Rhizocarpon geminatum on siliceous rock, C. Bay \& B. Fredskild, 4 August 1990.

Caloplaca cerina (Ehrh. ex Hedw.) Th. Fr. Germania Land, south west of Micardbu, $77^{\circ} 04^{\prime} \mathrm{N}$, $18^{\circ} 16^{\prime} \mathrm{W}$, on old bone together with Caloplaca tiroliensis, Candelariella aurella and Physcia dubia, C. Bay \& B. Fredskild, 17 August 1990; Schnauders $\varnothing, 78^{\circ} 52^{\prime} \mathrm{N}, 19^{\circ} 34^{\prime} \mathrm{W}$, on plant remains together with Caloplaca tiroliensis, C. Bay \& B. Fredskild, 9 August 1990.

* Caloplaca tiroliensis Zahlbr. - Germania Land, south west of Micardbu, $77^{\circ} 04^{\prime} \mathrm{N}, 18^{\circ} 16^{\prime} \mathrm{W}$, on old bone, C. Bay \& B. Fredskild, 17 August 1990; 
Schnauders $\varnothing, 78^{\circ} 52^{\prime} \mathrm{N}, 19^{\circ} 34^{\prime} \mathrm{W}$, on plant remains, C. Bay \& B. Fredskild, 9 August 1990.

Candelariella aurella (Hoffm.) Zahlbr. - Germania Land, south west of Micardbu, $77^{\circ} 04^{\prime} \mathrm{N}$, $18^{\circ} 16^{\prime} \mathrm{W}$, on old bone, C. Bay \& B. Fredskild, 17 August 1990.

Candelariella canadensis H. Magn. - Mørkefjord, c. $76^{\circ} 57^{\prime} \mathrm{N}, 20^{\circ} 30^{\prime} \mathrm{W}$, B. Lauritsen, $3 \mathrm{Au}-$ gust 1979; Danmarkshavn, $76^{\circ} 46^{\prime} \mathrm{N}, 18^{\circ} 36^{\prime} \mathrm{W}$, on soil, B. Lauritsen, 10 August 1981; Lambert Land, $79^{\circ} 08^{\prime} \mathrm{N}, 20^{\circ} 40^{\prime} \mathrm{W}$, alt. 125 m, C. Bay \& B. Fredskild, 9 August 1990.

Candelariella vitellina (Hoffm.) Müll. Arg. - Nr. Mellemland, $78^{\circ} 30^{\prime} \mathrm{N}, 21^{\circ} 10^{\prime} \mathrm{W}$, on siliceous rock, C. Bay \& B. Fredskild, 4 August 1990.

* Catapyrenium cinereum (Pers.) Körb. Schnauders $\varnothing, 78^{\circ} 52^{\prime} \mathrm{N}, 19^{\circ} 34^{\prime} \mathrm{W}$, on mineral soil, C. Bay \& B. Fredskild, 9 August 1990.

* Cephalophysis leucospila (Anzi) H. Kilias \& Scheid. - Lambert Land, $79^{\circ} 08^{\prime} \mathrm{N}, 20^{\circ} 40^{\prime} \mathrm{W}$, alt. $125 \mathrm{~m}$, on alkaline rock, C. Bay \& B. Fredskild, 9 August 1990.

Cetraria aculeata (Schreb.) Fr. - Danmarkshavn, $76^{\circ} 46^{\prime} \mathrm{N}, 18^{\circ} 36^{\prime} \mathrm{W}$, on soil, B. Lauritsen, 2 June, \& 28 August 1981; Germania Land, Flade Bugt, c. $77^{\circ} 15^{\prime} \mathrm{N}, 19^{\circ} 30^{\prime} \mathrm{W}$, alt. $210 \mathrm{~m}$, on soil, B. Lauritsen, 4 May 1982.

Cetraria islandica (L.) Ach. - Danmarkshavn, $76^{\circ} 46^{\prime} \mathrm{N}, 18^{\circ} 36^{\prime} \mathrm{W}$, on soil, B. Lauritsen, 29 June \& 28 August 1981 ; De Norske Øer, $79^{\circ} 03^{\prime} \mathrm{N}, 18^{\circ} 05^{\prime} \mathrm{W}$, alt. 150 m, on soil, C. Bay \& B. Fredskild, 4 August 1990.

* Cetrariella delisei (Bory ex Schaer.) Kärnefelt \& Thell - Danmarkshavn, $76^{\circ} 46^{\prime} \mathrm{N}, 18^{\circ} 36^{\prime} \mathrm{W}$, on soil, B. Lauritsen, 25 February \& 10 June 1981; Hvalrosodden, $76^{\circ} 57^{\prime} \mathrm{N}, 20^{\circ} 02^{\prime} \mathrm{W}$, on soil, B. Lauritsen, 11 July 1981; Germania Land, south west of Micardbu, $77^{\circ} 04^{\prime} \mathrm{N}, 18^{\circ} 16^{\prime} \mathrm{W}$, alt. $150 \mathrm{~m}$, on soil, C. Bay \& B. Fredskild, 17 August 1990; Stormlandet, north of Depotnæs, $77^{\circ} 36^{\prime} \mathrm{N}, 18^{\circ} 57^{\prime} \mathrm{W}$, alt. $50 \mathrm{~m}$, C. Bay \& B. Fredskild, 20 August 1990; Schnauders Ø, $78^{\circ} 52^{\prime} \mathrm{N}, 19^{\circ} 34^{\prime} \mathrm{W}$, on soil, C. Bay \& B. Fredskild, 9 August 1990.

* Cladonia borealis S. Stenroos - Danmarkshavn, $76^{\circ} 46^{\prime} \mathrm{N}, 18^{\circ} 36^{\prime} \mathrm{W}$, on soil, B. Lauritsen, $28 \mathrm{Au}-$ gust 1981; Stormlandet, north of Depotnæs, $77^{\circ} 36^{\prime} \mathrm{N}$, $18^{\circ} 57^{\prime} \mathrm{W}$, alt. $50 \mathrm{~m}$, on soil, C. Bay \& B. Fredskild, 20 August 1990; De Franske Øer, 7850’ N, $18^{\circ} 12^{\prime} \mathrm{W}$, alt. $50 \mathrm{~m}$, on soil, C. Bay \& B. Fredskild, 5 August 1990.

Cladonia fimbriata (L.) Fr. - Danmarkshavn, $76^{\circ} 46^{\prime} \mathrm{N}, 18^{\circ} 36^{\prime} \mathrm{W}$, on moss tussock together with Sphaerophorus fragilis and Stereocaulon alpinum, B. Lauritsen, 9 July 1982.

* Cladonia macroceras (Delise) Hav. - Danmarkshavn, $76^{\circ} 46^{\prime} \mathrm{N}, 18^{\circ} 36^{\prime} \mathrm{W}$, alt. $50 \mathrm{~m}$, on soil, B. Lauritsen, 29 July 1981.

* Cladonia pocillum (Ach.) Grognot - Mørkefjord, c. $76^{\circ} 57^{\prime} \mathrm{N}, 20^{\circ} 30^{\prime} \mathrm{W}$, on soil together with Dryas sp. and Lecanora epibryon, B. Lauritsen, 28 May 1981; Germania Land, south west of Micar$\mathrm{dbu}, 7^{\circ} 04^{\prime} \mathrm{N}, 18^{\circ} 16^{\prime} \mathrm{W}$, on soil, C. Bay \& B. Fredskild, 17 August 1990; De Franske Øer, 78³7'N, $18^{\circ} 35^{\prime} \mathrm{W}$, alt. $125 \mathrm{~m}$, on soil, C. Bay \& B. Fredskild, 5 August 1990.

Cladonia pyxidata (L.) Hoffm. - Mørkefjord, c. $76^{\circ} 57^{\prime} \mathrm{N}, 20^{\circ} 30^{\prime} \mathrm{W}$, on soil, B. Lauritsen, 28 July 1986.

* Cladonia trassii Ahti - Danmarkshavn, $76^{\circ} 46^{\prime} \mathrm{N}, 18^{\circ} 36^{\prime} \mathrm{W}$, on soil together with Cladonia borealis and C. pyxidata, B. Lauritsen, 29 July \& 28 August 1981.

Dactylina ramulosa (Hook.) Tuck. - Danmarkshavn, $76^{\circ} 46^{\prime} \mathrm{N}, 18^{\circ} 36^{\prime} \mathrm{W}$, on soil, B. Lauritsen, 12 July 1982 \& 28 July 1986. LGE 289.

* Dimelaena oreina (Ach.) Norman - V. Clausen Fjord, $77^{\circ} 30^{\prime} \mathrm{N}, 20^{\circ} 34^{\prime} \mathrm{W}$, alt. $50 \mathrm{~m}$, on rock composed of sandstone together with Pseudephebe minuscula and Sporastatia testudinea, C. Bay \& B. Fredskild, 15 August 1990; Nr. Mellemland, 78³0’ N, $21^{\circ} 10^{\prime} \mathrm{W}$, alt. $125 \mathrm{~m}$, on siliceous rock together with Umbilicaria virginis, C. Bay \& B. Fredskild, 4 August 1990.

Flavocetraria cucullata (Bellardi) Kärnefelt \& Thell - Schnauders $\varnothing, 78^{\circ} 52^{\prime} \mathrm{N}, 19^{\circ} 34^{\prime} \mathrm{W}$, alt. $100 \mathrm{~m}$, on soil, C. Bay \& B. Fredskild, 9 August 1990; Lambert Land, $79^{\circ} 08^{\prime} \mathrm{N}, 20^{\circ} 40^{\prime} \mathrm{W}$, alt. $125 \mathrm{~m}$, on soil, C. Bay \& B. Fredskild, 9 August 1990.

Flavocetraria nivalis (L.) Kärnefelt \& Thell Danmarkshavn, $76^{\circ} 46^{\prime} \mathrm{N}, 18^{\circ} 36^{\prime} \mathrm{W}$, on soil, B. Lauritsen, 21 March \& 10 June 1981; Germania Land, south west of Micardbu, $77^{\circ} 04^{\prime} \mathrm{N}, 18^{\circ} 16^{\prime} \mathrm{W}$, on soil, C. Bay \& B. Fredskild, 17 August 1990; V. Clausen Fjord, $77^{\circ} 30^{\prime} \mathrm{N}, 20^{\circ} 34^{\prime} \mathrm{W}$, alt. $50 \mathrm{~m}$, on soil, C. Bay \& B. Fredskild, 15 August 1990; De Franske Øer, $78^{\circ} 37^{\prime} \mathrm{N}, 18^{\circ} 35^{\prime} \mathrm{W}$, alt. $125 \mathrm{~m}, \mathrm{C}$. Bay \& B. Fredskild, 
5 August 1990; De Norske Øer, $79^{\circ} 03^{\prime} \mathrm{N}, 18^{\circ} 05^{\prime} \mathrm{W}$, alt. 150 m, C. Bay \& B. Fredskild, 4 August 1990; Lambert Land, Jørgen Brønlunds Grav, $79^{\circ} 12^{\prime} \mathrm{N}$, $19^{\circ} 07^{\prime} \mathrm{W}$, alt. $10 \mathrm{~m}$, on soil, C. Bay \& B. Fredskild, 4 August 1990.

* Fulgensia bracteata (Hoffm.) Räsänen - Nr. Mellemland, $78^{\circ} 30^{\prime} \mathrm{N}, 21^{\circ} 10^{\prime} \mathrm{W}$, alt. $75 \mathrm{~m}$, on soil, C. Bay \& B. Fredskild, 4 August 1990; Schnauders Ø, $78^{\circ} 52^{\prime} \mathrm{N}, 19^{\circ} 34^{\prime} \mathrm{W}$, alt. $100 \mathrm{~m}$, on soil, C. Bay \& B. Fredskild, 9 August 1990.

* Fuscopannaria praetermissa (Nyl.) P. M. Jørg. Nr. Mellemland, $78^{\circ} 30^{\prime} \mathrm{N}, 21^{\circ} 10^{\prime} \mathrm{W}$, alt. $125 \mathrm{~m}$, on mineral soil, C. Bay \& B. Fredskild, 4 August 1990.

Gowardia nigricans (Ach.) P. Halonen, L. Myllus, S. Velmela \& H. Hyvärinen - Danmarkshavn, $76^{\circ} 46^{\prime} \mathrm{N}, 18^{\circ} 36^{\prime} \mathrm{W}$, on soil, B. Lauritsen, 12 June \& 10 August 1981; De Norske Øer, $79^{\circ} 03^{\prime} \mathrm{N}, 18^{\circ} 05^{\prime} \mathrm{W}$, alt. 150 m, on soil, C. Bay \& B. Fredskild, 4 August 1990.

* Lecanora argopholis (Ach.) Ach. - Danmarkshavn, $76^{\circ} 46^{\prime} \mathrm{N}, 18^{\circ} 36^{\prime} \mathrm{W}$, on manured siliceous rock together with Lecanora polytropa and Xanthoria elegans, B. Lauritsen, 25 March 1981.

* Lecanora epibryon (Ach.) Ach. - Schnauders Ø, $78^{\circ} 52^{\prime} \mathrm{N}, 19^{\circ} 34^{\prime} \mathrm{W}$, alt. $100 \mathrm{~m}$, on soil, C. Bay \& B. Fredskild, 9 August 1990.

* Lecanora geophila (Th. Fr.) Poelt - Danmarkshavn, $76^{\circ} 46^{\prime} \mathrm{N}, 18^{\circ} 36^{\prime} \mathrm{W}$, on soil, B. Lauritsen, 10 August 1981; V. Clausen Fjord, $77^{\circ} 30^{\prime} \mathrm{N}, 20^{\circ} 34^{\prime} \mathrm{W}$, alt. 50 m, C. Bay \& B. Fredskild, 15 August 1990.

Lecanora hagenii (Ach.) Ach. v. fallax Hepp Danmarkshavn, $76^{\circ} 46^{\prime} \mathrm{N}, 18^{\circ} 36^{\prime} \mathrm{W}$, on old bone together with Lecidella euphorea, B. Lauritsen, 12 June 1981.

* Lecanora intricata (Ach.) Ach. - Lambert Land, $79^{\circ} 08^{\prime} \mathrm{N}, 20^{\circ} 40^{\prime} \mathrm{W}$, alt. $125 \mathrm{~m}$, on siliceous rock, C. Bay \& B. Fredskild, 9 August 1990.

Lecanora marginata (Schaer.) Hertel \& Rambold Nr. Mellemland, $78^{\circ} 30^{\prime} \mathrm{N}, 21^{\circ} 10^{\prime} \mathrm{W}$, alt. $125 \mathrm{~m}$, on siliceous rock, C. Bay \& B. Fredskild, 4 August 1990; Lambert Land, $79^{\circ} 08^{\prime} \mathrm{N}, 20^{\circ} 40^{\prime} \mathrm{W}$, alt. $125 \mathrm{~m}$, on alkaline rock, 9 August 1990.

Lecanora polytropa (Ehrh. ex Hoffm.) Rabenh. - Godfred Hansens $\varnothing$, c. $75^{\circ} 25^{\prime} \mathrm{N}, 21^{\circ} 00^{\prime} \mathrm{W}$, on siliceous rock, B. Lauritsen, 1 \& 3 April 1981; Danmarkshavn, $76^{\circ} 46^{\prime} \mathrm{N}, 18^{\circ} 36^{\prime} \mathrm{W}$, on siliceous rock, B. Lauritsen, 25 March 1981.

Lecidea atrobrunnea (Ramond ex Lam. \& DC.)
Schaer. - Godfred Hansens $\varnothing$, c. $75^{\circ} 25^{\prime} \mathrm{N}, 21^{\circ} 00^{\prime} \mathrm{W}$, on siliceous rock, B. Lauritsen, 3 April 1981; Danmarkshavn, $76^{\circ} 46^{\prime} \mathrm{N}, 18^{\circ} 36^{\prime \prime} \mathrm{W}$, on siliceous rock, B. Lauritsen, 25 March 1981.

* Lecidea lapicida (Ach.) Ach. v. pantherina Ach. - Danmarkshavn, $76^{\circ} 46^{\prime} \mathrm{N}, 18^{\circ} 36^{\prime} \mathrm{W}$, on siliceous rock, B. Lauritsen, 2 June 1981.

* Lecidea ramulosa Th. Fr. - Schnauders Ø, $78^{\circ} 52^{\prime} \mathrm{N}, 19^{\circ} 34^{\prime} \mathrm{W}$, on soil together with Solorina bispora, C. Bay \& B. Fredskild, 9 August 1990.

* Lecidea tesselata Flörke - Nr. Mellemland, $78^{\circ} 30^{\prime \prime} \mathrm{N}, 21^{\circ} 10^{\prime} \mathrm{W}$, alt. $125 \mathrm{~m}$, on siliceous rock together with Xanthoria elegans, Rhizocarpon geminatum and Sporastatia testudinea, C. Bay \& B. Fredskild, 4 August 1990.

* Lecidella euphorea (Flörke) Hertel - Danmarkshavn, $76^{\circ} 46^{\prime} \mathrm{N}, 18^{\circ} 36^{\prime} \mathrm{W}$, on old bone, B. Lauritsen, 12 June 1981.

* Leprocaulon subalbicans (I. M. Lamb) I. M. Lamb \& A. M. Ward - De Franske Øer, $78^{\circ} 37^{\prime} \mathrm{N}, 18^{\circ} 35^{\prime} \mathrm{W}$, alt. $125 \mathrm{~m}$, over mosses on soil, C. Bay \& B. Fredskild, 5 August 1990.

Melanelia stygia (L.) Essl. - Danmarkshavn, $76^{\circ} 46^{\prime} \mathrm{N}, 18^{\circ} 36^{\prime} \mathrm{W}$, on siliceous rock, B. Lauritsen, 13 June \& 10 August 1981.

* Melanohalea elegantula (Zahlbr.) O. Blanko, A. Crespo, Divakar, Essl., D. Hawksw. \& Lumbsch - Godfred Hansens $\varnothing, 75^{\circ} 25^{\prime} \mathrm{N}, 21^{\circ} 00^{\prime} \mathrm{W}$, on siliceous rock together with Umbilicaria krascheninnikovii, B. Lauritsen, 1 April 1981; Stormlandet, Kap Amélie, c. $77^{\circ} 33^{\prime} \mathrm{N}, 19^{\circ} 25^{\prime} \mathrm{W}$, alt. 190 m, on manured siliceous rock, B. Lauritsen, 1 May 1981; Lambert Land, $79^{\circ} 08^{\prime} \mathrm{N}, 20^{\circ} 40^{\prime} \mathrm{W}$, alt. $125 \mathrm{~m}$, C. Bay \& B. Fredskild, 9 August 1990.

* Peltigera leucophlebia (Nyl.) Gyeln. - Mørkefjord, c. $76^{\circ} 57^{\prime} \mathrm{N}, 20^{\circ} 30^{\prime} \mathrm{W}$, on soil in peat shed, B. Lauritsen, 3 August 1979; Stormlandet, north of Depotnæs, $77^{\circ} 36^{\prime} \mathrm{N}, 18^{\circ} 57^{\prime} \mathrm{W}$, alt. $50 \mathrm{~m}$, on soil, C. Bay \& B. Fredskild, 20 August 1990; De Franske Øer, $78^{\circ} 37^{\prime} \mathrm{N}, 18^{\circ} 35^{\prime \prime} \mathrm{W}$, alt. $125 \mathrm{~m}$, on soil, C. Bay \& B. Fredskild, 5 August 1990.

* Peltigera lepidophora (Nyl. ex Vain.) Bitter Danmarkshavn, $76^{\circ} 46^{\prime} \mathrm{N}, 18^{\circ} 36^{\prime} \mathrm{W}$, on soil, B. Lauritsen, 10 August 1981.

Peltigera rufescens (Weiss) Humb. - Lambert Land, $79^{\circ} 08^{\prime} \mathrm{N}, 20^{\circ} 40^{\prime} \mathrm{W}$, alt. $125 \mathrm{~m}$, on soil, C. Bay \& B. Fredskild, 9 August 1990.

* Phaeorrhiza nimbosa (Fr.) H. Mayrhofer \& Po- 
elt - Danmarkshavn, $76^{\circ} 46^{\prime} \mathrm{N}, 18^{\circ} 36^{\prime} \mathrm{W}$, on mineral soil, B. Lauritsen, 10 August 1981.

Physcia dubia (Hoffm.) Lettau - Germania Land, south west of Micardbu, $77^{\circ} 04^{\prime} \mathrm{N}, 18^{\circ} 16^{\prime} \mathrm{W}$, on old bone, C. Bay \& B. Fredskild, 17 August 1990; Lambert Land, $79^{\circ} 08^{\prime} \mathrm{N}, 20^{\circ} 40^{\prime} \mathrm{W}$, alt. $125 \mathrm{~m}$, on manured siliceous rock, C. Bay \& B. Fredskild, 9 August 1990.

Physconia muscigena (Ach.) Poelt - Danmarkshavn, $76^{\circ} 46^{\prime} \mathrm{N}, 18^{\circ} 36^{\prime \prime} \mathrm{W}$, on soil, B. Lauritsen, 25 March \& 10 August 1981; V. Clausen Fjord, $77^{\circ} 30^{\prime} \mathrm{N}, 20^{\circ} 34^{\prime} \mathrm{W}$, alt. $50 \mathrm{~m}$, on soil, C. Bay \& B. Fredskild, 15 August 1990; Schnauders Ø, $78^{\circ} 52^{\prime} \mathrm{N}, 19^{\circ} 34^{\prime} \mathrm{W}$, on soil C. Bay \& B. Fredskild, 9 August 1990; Lambert Land, 7908' N, 2040”W, alt. 125 m, on soil, C. Bay \& B. Fredskild, 9 August 1990.

* Placynthium asperellum (Ach.) Trevis. Schnauders $\varnothing, 78^{\circ} 52^{\prime} \mathrm{W}, 19^{\circ} 34^{\prime \prime} \mathrm{W}$, on siliceous rock, C. Bay \& B. Fredskild, 9 August 1990.

* Pleopsidium chlorophanum (Wahlenb.) Zopf Danmarkshavn, $76^{\circ} 46^{\prime} \mathrm{N}, 18^{\circ} 36^{\prime} \mathrm{W}$, on siliceous rock, B. Lauritsen, 29 August 1979.

* Protoblastenia terricola (Anzi) Lynge Schnauders $\varnothing, 78^{\circ} 52^{\prime} \mathrm{N}, 19^{\circ} 34^{\prime \prime} \mathrm{W}$, alt. $100 \mathrm{~m}$, on mineral soil, C. Bay \& B. Fredskild, 9 August 1990.

Pseudephebe minuscula (Nyl. ex Arnold) Brodo \& D. Hawksw. - Danmarkshavn, 76 46 ${ }^{\prime} \mathrm{N}, 18^{\circ} 36^{\prime} \mathrm{W}$, on siliceous rock, C. Bay \& B. Fredskild, 25 March 2 June \& 10 August 1981; Germania Land, south west of Micardbu, $77^{\circ} 04^{\prime} \mathrm{N}, 18^{\circ} 16^{\prime} \mathrm{W}$, alt. $125 \mathrm{~m}$, on siliceous stone, C. Bay \& B. Fredskild, $17 \mathrm{Au}-$

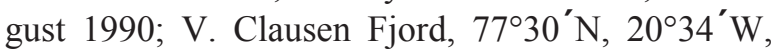
alt. $50 \mathrm{~m}$, on rock composed of sandstone, C. Bay \& B. Fredskild, 15 August 1990; Stormlandet, Kap Amélie, $77^{\circ} 33^{\prime} \mathrm{N}, 19^{\circ} 25^{\prime} \mathrm{W}$, alt. $190 \mathrm{~m}$, on siliceous rock, C. Bay \& B. Fredskild, 1 May 1981; Lambert Land, $79^{\circ} 08^{\prime} \mathrm{N}, 20^{\circ} 40^{\prime} \mathrm{W}$, alt. $125 \mathrm{~m}$, on siliceous rock, 9 August 1990.

Rhizocarpon geminatum Körb. - Danmarkshavn, $76^{\circ} 46^{\prime} \mathrm{N}, 18^{\circ} 36^{\prime} \mathrm{W}$, on siliceous rock, B. Lauritsen, 10 \& 12 June 1981; Stormlandet, north of Depotnæs, $77^{\circ} 36^{\prime} \mathrm{N}, 18^{\circ} 57^{\prime} \mathrm{W}$, alt. $50 \mathrm{~m}$, C. Bay \& B. Fredskild, 20 August 1990; Nr. Mellemland, $78^{\circ} 30^{\prime \prime} \mathrm{N}, 21^{\circ} 10^{\prime} \mathrm{W}$, alt. $125 \mathrm{~m}$, on siliceous rock, C. Bay \& B. Fredskild, 4 August 1990; Schnauders Ø, $78^{\circ} 52^{\prime} \mathrm{N}, 19^{\circ} 34^{\prime} \mathrm{W}$, on siliceous stones, C. Bay \& B. Fredskild, 9 August 1990.
Rhizocarpon geographicum (L.) DC. - Danmarkshavn, $76^{\circ} 46^{\prime} \mathrm{N}, 18^{\circ} 36^{\prime} \mathrm{W}$, on siliceous rock, B. Lauritsen, 25 February 2 June \& 10 August 1981; Germania Land, Flade Bugt, c. $77^{\circ} 15^{\prime} \mathrm{N}, 19^{\circ} 30^{\prime} \mathrm{W}$, alt. $210 \mathrm{~m}$, on siliceous rock, B. Lauritsen, 4 May 1981.

* Rhizocarpon jemtlandicum (Malme) Malme Danmarkshavn, $76^{\circ} 46^{\prime} \mathrm{N}, 18^{\circ} 36^{\prime} \mathrm{W}$, on siliceous rock, B. Lauritsen, 12 June 1981; Germania Land, Flade Bugt, c. $77^{\circ} 15^{\prime} \mathrm{N}, 19^{\circ} 30^{\prime} \mathrm{W}$, alt. $210 \mathrm{~m}$, on siliceous rock, B. Lauritsen, 4 May 1981.

* Rhizocarpon pusillum Runemark - V. Clausen Fjord, $77^{\circ} 30^{\prime} \mathrm{N}, 20^{\circ} 34^{\prime \prime} \mathrm{W}$, alt. $50 \mathrm{~m}$, on Sporastatia testudinea on siliceous rock, C. Bay \& B. Fredskild, 15 August 1990; Stormlandet, north of Depotnæs, $77^{\circ} 36^{\prime} \mathrm{N}, 18^{\circ} 57^{\prime} \mathrm{W}$, alt. $50 \mathrm{~m}$, on Sporastatia testudinea on siliceous rock, C. Bay \& B. Fredskild, $20 \mathrm{Au}-$ gust 1990; Nr. Mellemland, $78^{\circ} 30^{\prime} \mathrm{N}, 2^{\circ} 10^{\prime} \mathrm{W}$, alt. $125 \mathrm{~m}$, on Sporastatia testudinea on siliceous rock, C. Bay \& B. Fredskild, 4 August 1990.

Rhizoplaca melanophthalma (DC.) Leuckert \& Poelt - Godfred Hansens Ø, $75^{\circ} 25^{\prime} \mathrm{N}, 21^{\circ} 00^{\prime} \mathrm{W}$, on siliceous rock, B. Lauritsen, 1 \& 3 April 1981; Danmarkshavn, $76^{\circ} 46^{\prime \prime} \mathrm{N}, 18^{\circ} 36^{\prime} \mathrm{W}$, on siliceous rock, B. Lauritsen, 13 June 1981; Stormlandet, Kap Amélie, $77^{\circ} 33^{\prime} \mathrm{N}, 19^{\circ} 25^{\prime} \mathrm{W}$, alt. $190 \mathrm{~m}$, on manured siliceous rock, B. Lauritsen, May 1, 1981; Lambert Land, $79^{\circ} 08^{\prime} \mathrm{N}, 20^{\circ} 40^{\prime} \mathrm{W}$, alt. $125 \mathrm{~m}$, on siliceous rock, C. Bay \& B. Fredskild, 9 August 1990.

* Rinodina archaea (Ach.) Arnold - Danmarkshavn, $76^{\circ} 46^{\prime} \mathrm{N}, 18^{\circ} 36^{\prime} \mathrm{W}$, on soil, B. Lauritsen, 12 June 1981.

Rinodina mniaraea (Ach.) Körb. - Schnauders Ø, $78^{\circ} 52^{\prime} \mathrm{N}, 19^{\circ} 34^{\prime} \mathrm{W}$, alt. $100 \mathrm{~m}$, on soil, C. Bay \& B. Fredskild, 9 August 1990.

Solorina bispora Nyl. - Schnauders Ø, 78 $52^{\circ} \mathrm{N}$, $19^{\circ} 34^{\prime} \mathrm{W}$, alt. $100 \mathrm{~m}$, on soil, C. Bay \& B. Fredskild, 9 August 1990; Lambert Land, $79^{\circ} 08^{\prime} \mathrm{N}, 20^{\circ} 40^{\prime} \mathrm{W}$, alt. 125 m, on soil, C. Bay \& B. Fredskild, 9 August 1990.

Solorina crocea (L.) Ach. - Stormlandet, north of Depotnæs, $77^{\circ} 36^{\prime \prime} \mathrm{N}, 18^{\circ} 57^{\prime} \mathrm{W}$, alt. $50 \mathrm{~m}$, on soil, C. Bay \& B. Fredskild, 20 August 1990; De Franske Øer, $78^{\circ} 37^{\prime} \mathrm{N}, 18^{\circ} 35^{\prime} \mathrm{W}$, alt. $125 \mathrm{~m}$, on soil, C. Bay \& B. Fredskild, 5 August 1990.

* Sphaerophorus fragilis (L.) Pers. - Danmarkshavn, $76^{\circ} 46^{\prime} \mathrm{N}, 18^{\circ} 36^{\prime} \mathrm{W}$, on mosses, B. Lauritsen, 9 July 1982. 
Sporastatia testudinea (Ach.) A. Massal. - Germania Land, south west of Micardbu, $77^{\circ} 04^{\prime \prime} \mathrm{N}$, $18^{\circ} 16^{\prime} \mathrm{W}$, alt. $125 \mathrm{~m}$, on siliceous stone, C. Bay \& B. Fredskild, 17 August 1990; V. Clausen Fjord, $77^{\circ} 30^{\prime} \mathrm{N}, 20^{\circ} 34^{\prime \prime} \mathrm{W}$, alt. $50 \mathrm{~m}$, on rock composed of sandstone, C. Bay \& B. Fredskild, 15 August 1990; Stormlandet, north of Depotnæs, $77^{\circ} 36^{\prime} \mathrm{N}, 18^{\circ} 57^{\prime} \mathrm{W}$, alt. $50 \mathrm{~m}$, on siliceous rock, C. Bay \& B. Fredskild, 20 August 1990; Nr. Mellemland, $78^{\circ} 30^{\prime} \mathrm{N}$, $21^{\circ} 10^{\prime} \mathrm{W}$, alt. $125 \mathrm{~m}$, on siliceous rock, C. Bay \& B. Fredskild, 4 August 1990.

* Stereocaulon alpinum Laurer - Danmarkshavn, $76^{\circ} 46^{\prime} \mathrm{N}, 18^{\circ} 36^{\prime} \mathrm{W}$, on soil, B. Lauritsen, 10 June 1981 \& 9 July 1982.

* Stereocaulon arenarium (Savicz) I. M. Lamb Danmarkshavn, $76^{\circ} 46^{\prime} \mathrm{N}, 18^{\circ} 36^{\prime} \mathrm{W}$, on soil, B. Lauritsen, 28 August 1981; Nr. Mellemland, 78 $30^{\prime} \mathrm{N}$, $21^{\circ} 10^{\prime} \mathrm{W}$, alt. $75 \mathrm{~m}$, on soil, C. Bay \& B. Fredskild, 4 August 1990.

* Stereocaulon glareosum (Savicz) H. Magn. Danmarkshavn, 7646' N, 18³6'W, on gravely soil, B. Lauritsen, 28 August 1981; Stormlandet, north of Depotnæs, $77^{\circ} 36^{\prime} \mathrm{N}, 18^{\circ} 57^{\prime} \mathrm{W}$, alt. $50 \mathrm{~m}$, on soil, C. Bay \& B. Fredskild, 20 August 1990; De Franske Øer, $78^{\circ} 50^{\prime} \mathrm{N}, 18^{\circ} 12^{\prime} \mathrm{W}$, alt. $50 \mathrm{~m}, \mathrm{C}$. Bay \& B. Fredskild, 5 August 1990; Schnauders Ø, $78^{\circ} 52^{\prime} \mathrm{N}$, $19^{\circ} 34^{\prime} \mathrm{W}$, on gravely soil, C. Bay \& B. Fredskild, 9 August 1990; Lambert Land, $79^{\circ} 08^{\prime} \mathrm{N}, 20^{\circ} 40^{\prime} \mathrm{W}$, alt. 125 m, on soil, C. Bay \& B. Fredskild, 9 August 1990.

Thamnolia vermicularis (Sw.) Schaer. v. subuliformis (Ehrh.) Schaer. - Danmarkshavn, 76 $46^{\prime} \mathrm{N}$, $18^{\circ} 36^{\prime} \mathrm{W}$, on soil, B. Lauritsen, 12 \& 29 June \& 28 August 1981; Nr. Mellemland, 78³0'N, $21^{\circ} 10^{\prime} \mathrm{W}$, alt. $125 \mathrm{~m}$, on soil, C. Bay \& B. Fredskild, 4 August 1990; Schnauders Ø, 7852”N, 19³4”W, on soil, C. Bay \& B. Fredskild, 9 August 1990; Lambert Land, $79^{\circ} 08^{\prime} \mathrm{N}, 20^{\circ} 40^{\prime} \mathrm{W}$, alt. $125 \mathrm{~m}$, on soil, C. Bay \& B. Fredskild, 9 August 1990.

* Umbilicaria arctica (Ach.) Nyl. - Godfred Hansens $\varnothing, 75^{\circ} 25^{\prime} \mathrm{N}, 21^{\circ} 00^{\prime} \mathrm{W}$, on siliceous rock, B. Lauritsen, 1 \& 3 April 1981.

Umbilicaria decussata (Vill.) Zahlbr. - Godfred Hansens $\varnothing, 75^{\circ} 25^{\prime} \mathrm{N}, 21^{\circ} 00^{\prime} \mathrm{W}$, on siliceous rock, B. Lauritsen, 1 April 1981; Danmarkshavn, $76^{\circ} 46^{\prime} \mathrm{N}, 18^{\circ} 36^{\prime} \mathrm{W}$, on siliceous rock, B. Lauritsen, 23 February 1981; Kap Amélie, $77^{\circ} 33^{\prime} \mathrm{N}, 19^{\circ} 25^{\prime} \mathrm{W}$, alt. $190 \mathrm{~m}$, on siliceous rock, B. Lauritsen, 1 May
1981; Lambert Land, $79^{\circ} 08^{\prime} \mathrm{N}, 20^{\circ} 40^{\prime} \mathrm{W}$, alt. $125 \mathrm{~m}$, on siliceous rock, C. Bay \& B. Fredskild, 9 August 1990.

Umbilicaria hyperborea (Ach.) Hoffm. - Danmarkshavn, $76^{\circ} 46^{\prime} \mathrm{N}, 18^{\circ} 36^{\prime} \mathrm{W}$, on siliceous rock, B. Lauritsen, 25 March \& 12 June 1981.

* Umbilicaria krascheninnikovii (Savicz) Zahlbr. Godfred Hansens $\varnothing, 75^{\circ} 25^{\prime} \mathrm{N}, 21^{\circ} 00^{\prime} \mathrm{W}$, on siliceous rock, B. Lauritsen, 1 April 1981.

* Umbilicaria leiocarpa DC. - Godfred Hansens $\varnothing, 75^{\circ} 25^{\prime} \mathrm{N}, 21^{\circ} 00^{\prime} \mathrm{W}$, on siliceous rock, B. Lauritsen, 3 April 1981; Danmarkshavn, $76^{\circ} 46^{\prime} \mathrm{N}, 18^{\circ} 36^{\prime} \mathrm{W}$, on siliceous rock, B. Lauritsen, 24 July 1981; De Norske Øer, $79^{\circ} 03^{\prime \prime} \mathrm{N}, 18^{\circ} 05^{\prime} \mathrm{W}$, alt. $150 \mathrm{~m}$, on siliceous rock, C. Bay \& B. Fredskild, 4 August 1990.

* Umbilicaria lyngei Schol. - Danmarkshavn, $76^{\circ} 46^{\prime} \mathrm{N}, 18^{\circ} 36^{\prime} \mathrm{W}$, on siliceous rock, (who?) 25 February \& 29 July 1981; Germania Land, south west of Micardbu, $77^{\circ} 04^{\prime} \mathrm{N}, 18^{\circ} 16^{\prime} \mathrm{W}$, on siliceous rock, C. Bay \& B. Fredskild, 17 August 1990; Kap Amélie, $77^{\circ} 33^{\prime} \mathrm{N}, 19^{\circ} 25^{\prime} \mathrm{W}$, alt. $190 \mathrm{~m}$, on siliceous rock, B. Lauritsen, 1 May 1981; Stormlandet, north of Depotnæs, $77^{\circ} 36^{\prime} \mathrm{N}, 18^{\circ} 57^{\prime} \mathrm{W}$, alt. $50 \mathrm{~m}$, on siliceous rock, C. Bay \& B. Fredskild, 20 August 1990.

Umbilicaria proboscidea (L.) Schrad. - Danmarkshavn, $76^{\circ} 46^{\prime} \mathrm{N}, 18^{\circ} 36^{\prime} \mathrm{W}$, on siliceous rock, B. Lauritsen, 10 June \& 25 July 1981. LGE 288.

Umbilicaria torrefacta (Lightf.) Schrad. - Danmarkshavn, $76^{\circ} 46^{\prime} \mathrm{N}, 18^{\circ} 36^{\prime} \mathrm{W}$, on siliceous rock, B. Lauritsen, 10 June \& 28 August 1981.

* Umbilicaria vellea (L.) Hoffm. - Danmarkshavn, $76^{\circ} 46^{\prime} \mathrm{N}, 18^{\circ} 36^{\prime} \mathrm{W}$, on siliceous stone, B. Lauritsen, 21 March 1981.

Umbilicaria virginis Schaer. - Godfred Hansens Ø, $75^{\circ} 25^{\prime} \mathrm{N}, 21^{\circ} 00^{\prime} \mathrm{W}$, on siliceous rock, B. Lauritsen, 1 April 1981; Danmarkshavn, $76^{\circ} 46^{\prime} \mathrm{N}, 18^{\circ} 36^{\prime} \mathrm{W}$, on siliceous rock, (who?) 13 April \& 10 June 1981; Hvalrosodden, $76^{\circ} 57^{\prime \prime} \mathrm{N}, 20^{\circ} 02^{\prime \prime} \mathrm{W}$, on siliceous rock, B. Lauritsen, 11 July 1981; V. Clausen Fjord, $77^{\circ} 30^{\prime} \mathrm{N}$, $20^{\circ} 34^{\prime} \mathrm{W}$, alt. $50 \mathrm{~m}, \mathrm{C}$. Bay \& B. Fredskild, $15 \mathrm{Au}-$ gust 1990; Stormlandet, north of Depotnæs, $77^{\circ} 36^{\prime} \mathrm{N}$, $18^{\circ} 57^{\prime} \mathrm{W}$, alt $50 \mathrm{~m}$, on siliceous rock, C. Bay \& B. Fredskild, 20 August 1990; Nr. Mellemland, 78³0'N, $21^{\circ} 10^{\prime} \mathrm{W}$, alt. $125 \mathrm{~m}$, on siliceous rock, C. Bay \& B. Fredskild, 4 August 1990; Lambert Land, Jørgen Brønlunds Grav, $79^{\circ} 12^{\prime} \mathrm{N}, 19^{\circ} 07^{\prime} \mathrm{W}$, on siliceous rock, C. Bay \& B. Fredskild, 4 August 1990. LGE 286.

Usnea sphacelata R. Br. - Danmarkshavn, 
$76^{\circ} 46^{\prime} \mathrm{N}, 18^{\circ} 36^{\prime} \mathrm{W}$, on siliceous rock, B. Lauritsen, 12 June \& 28 August 1981; Germania Land, south west of Micardbu, $77^{\circ} 04^{\prime} \mathrm{N}, 18^{\circ} 16^{\prime} \mathrm{W}$, on siliceous rock, C. Bay \& B. Fredskild, 17 August 1990. LGE 287.

Xanthoria candelaria (L.) Th. Fr. - Lambert Land, $79^{\circ} 08^{\prime} \mathrm{N}, 20^{\circ} 40^{\prime} \mathrm{W}$, alt. $125 \mathrm{~m}$, on siliceous rock, C. Bay \& B. Fredskild, 9 August 1990.

Xanthoria elegans (Link) Th. Fr. - Danmarkshavn, $76^{\circ} 46^{\prime} \mathrm{N}, 18^{\circ} 36^{\prime} \mathrm{W}$, on siliceous rock, B. Lauritsen, 21 March 1981; V. Clausen Fjord, 77³0'N, $20^{\circ} 34^{\prime} \mathrm{W}$, alt. $50 \mathrm{~m}$, on siliceous rock, C. Bay \& B. Fredskild, 15 August 1990; Nr. Mellemland, $78^{\circ} 30^{\prime} \mathrm{N}, 21^{\circ} 10^{\prime \prime} \mathrm{W}$, alt. $125 \mathrm{~m}$, on siliceous rock, C. Bay \& B. Fredskild, 4 August 1990; Lambert Land, $79^{\circ} 08^{\prime} \mathrm{N}, 20^{\circ} 40^{\prime} \mathrm{W}$, alt. $125 \mathrm{~m}$, on siliceous rock, C. Bay \& B. Fredskild, 9 August 1990.

The Denmark Expedition resulted in a great increase in our knowledge on the lichen flora of the most northern regions of East Greenland, and this progress was continued by many new expeditions to the polar desert during the following hundred years. However, the logistics is expensive and often intricate in these uninhabited areas, and, therefore, some of them still must be considered incompletely known as regards the lichens. The collections dealt with in the present paper give an impression of a well-developed fell-field vegetation with common and widely distributed species such as Bryocaulon divergens, Bryoria chalybeiformis, Cetraria aculeata, Flavocetraria nivalis, Gowardia nigricans and Thamnolia vermicularis v. subuliformis. The group of lichens with a particular preference for sligtly alkaline soil consists of the following species: Acarospora schleicheri, Buellia elegans, Catapyrenium cinereum, Cladonia pocillum, Fulgensia bracteata, Fuscopannaria praetermissa, Lecanora epibryon, Peltigera rufescens, Phaeorrhiza nimbosa, Physconia muscigena, Protoblastenia terricola and Solorina bispora. These lichens occur on steppe-like slopes, in open Dryas heaths and in fell-fields predominantly in more or less continental areas (HANSEN, 1995, 2000). Arctocetraria nigricascens is a comparatively rare lichen with a northern distribution in Greenland. It often grows together with Cetrariella delisei in moist patches in open dwarf shrub heaths (HANSEN, 2011). The group of saxicolous lichens is rather heteroge- nous and consists of many nitrophilous species such as Dimelaena oreina, Melanohalea elegantula, Rhizoplaca melanophthalma and Umbilicaria decussata and species without specific demands as regards the substrate, for example Pseudephebe minuscula and some Umbilicarias. This group also includes a few lichens with a particular preference for calcareous rocks, for example, Cephalophysis leucospila. No doubt the saxicolous lichens are under-represented in the collections, so it is an important task for lichenologists in these rough, but intriguing areas.

\section{ACKNOWLEDGEMENTS}

I wish to thank Christian Bay, Bent Fredskild and Børge Lauritsen for handing over their lichen collections to me for identification.

\section{REFERENCES}

Alstrup V., Hansen E. S., Daniels F. J. A., 2000: Lichenized, lichenicolous and other fungi from North and North-East Greenland. - Folia Cryptogamica Estonica, 37: 1-20.

BRANTH J. D., 1894: Lichener fra Scoresby Sund og Hold with Hope. - Meddelelser om Grønland, 3: 84-103.

Escher A., Stuart Watt W., 1976: Geology of Greenland. - Copenhagen.

FredSKILD B., BAY C., 1991: Grønlands botaniske undersøgelse 1990. Teknisk Rapport. - Copenhagen.

Galløe O., 1910: Lichens from North-East Greenland (N. of $76^{\circ} \mathrm{N}$. lat.) collected by the 'DanmarkExpedition' 1906-1908. - Meddelelser om Grønland, 43: 181-191.

HANSEN E. S., 1982: Lichens from Central East Greenland. - Meddelelser om Grønland. Bioscience, 9: 1-33.

HANSEn E. S., 1995: The lichen flora of the Jørgen Brønlund fjord area, northern Greenland. - Bibliotheca Lichenologica, 57: 187-198.

Hansen E. S., 2000: A contribution to the lichen flora of the Kangerlussuaq area, West Greenland. Cryptogamie, Mycologie, 21: 53-59.

Hansen E. S., 2006: 3.3 Lichens. - In: KlitgaARd A. B., Rasch M., CANing K. (eds), Zacken- 
beg Ecological Research Operations, $11^{\text {th }}$ Annual Report 2005. Technical Report. - Copenhagen.

HANSEN E. S., 2008a: A contribution to the lichen flora of the Scoresby Sund area, Central East Greenland. - Cryptogamie, Mycologie, 29: 293-302.

HANSEN E. S., 2008b: The lichen flora of Kuhn Ø and Blåbærdalen, easternmost Th. Thomsen Land, North East Greenland. - Cryptogamie, Mycologie, 29: 397-411.

HANSEN E. S., 2009: A contribution to the lichen flora of Hold with Hope and Hudson Land, North East Greenland. - Cryptogamie, Mycologie, 30: 269-278.

HANSEn E. S., 2010: A contribution to the lichen flora of Scoresby Land, Central East Greenland. Cryptogamie, Mycologie, 31: 453-462.
HANSEn E. S., 2011: Lichens from Qaanaaq and Siorapaluk, North West Greenland. - Folia Cryptogamica Estonica, 48: 5-11.

HARTz N., 1895: Østgrønlands Vegetationsforhold. Meddelelser om Grønland, 18: 105-314.

Jensen B. (ed.), 1999: Grønlands Biodiversitet - et landestudie. Teknisk Rapport, 27. - Nuuk.

Lynge B., 1940: Lichens from North East Greenland II. Microlichens. - In: Skrifter om Svalbard og Ishavet, 81: 1-143.

Lynge B., Scholander P. F., 1932: Lichens from North East Greenland. I. - In: Skrifter om Svalbard og Ishavet, 41: 1-116.

Santesson R., Moberg R., Nordin A., Tønsberg T., VitiKAINEN O., 2004: Lichen-forming and lichenicolous fungi of Fennoscandia. - Uppsala.

\section{PAPILDOMI DUOMENYS APIE ŠIAURĖS RYTŲ GRENLANDIJOS FLORĄ}

\section{Eric Steen Hansen}

\section{Santrauka}

Straipsnyje pateikiamas 83 rūšių kerpių sąrašas. Kerpès surinktos šiaurès rytinejje Grenlandijoje, $\operatorname{tarp} 75^{\circ}$ and $80^{\circ}$ šiaurès platumos. Kiek daugiau nei pusè sąrašo rūšių (45) aptiktos pirmą kartą šiame regione. Pateiktoje medžiagoje ypač gerai atstovaujamos tundros šlaitų bendrijoms būdingos kerpès, augančios tiek ant šarmingų, tiek neutralių ir rūgščiu dirvožemių, tačiau yra ir rūšiu augančių drègnuose krūmokšniais apaugusiuose ploteliuose bei ploteliuose su ilgiau išsilaikančiu sniegu. Sąraše taip pat nemažai epilitinių kerpių, augančių ant silikatinių, tiek paukščių ekskrementų veikiamų, tiek ir guano nepaveiktų uolienų, taip pat ir kai kurių šarminių uolienų. 